\title{
Normas de Emocionalidade para a Versão Brasileira do Paradigma Deese-Roediger-McDermott (DRM) ${ }^{1}$
}

\author{
Renato Favarin dos Santos \\ Universidade Federal de Roraima \\ Ronie Alexsandro Teles da Silveira \\ Universidade Federal do Recôncavo da Bahia \\ Carlos Falcão de Azevedo Gomes \\ Lilian Milnitsky Stein ${ }^{2}$ \\ Pontificia Universidade Católica do Rio Grande do Sul
}

\begin{abstract}
RESUMO - A necessidade de se estabelecer normas de emocionalidade para material verbal de idioma português-brasileiro se origina da ausência de padrões para a incipiente pesquisa sobre emoção. O presente estudo teve por objetivo obter medidas de emocionalidade para a versão brasileira do paradigma Deese-Roediger-McDermott (DRM). Um total de 516 universitários avaliou a valência e o alerta de 44 listas de palavras semanticamente associadas e suas palavras críticas. A análise de confiabilidade interna das normas de emocionalidade mostrou correlações altas. Os resultados indicaram a possibilidade da utilização da versão brasileira do DRM em pesquisas sobre emoção e cognição.
\end{abstract}

Palavras-chave: normas; emoção; alerta; valência; palavras associadas.

\section{Emotionality Norms for the Brazilian Version of the Deese-Roediger-McDermott (DRM) Paradigm}

\begin{abstract}
Establishing emotionality norms for verbal material in Portuguese is a relevant methodological contribution to the emergent research on emotion. The present study aimed at obtaining measures of emotionality for the Brazilian version of the Deese-Roediger-McDermott (DRM) paradigm. A total of 516 undergraduate students rated 44 lists of words associated by semantics and their critical words with respect to valence and arousal. The reliability analysis of the emotionality norms showed high correlations. The results indicate the adequacy of using the Brazilian version of the DRM paradigm for the study of emotion and cognition.
\end{abstract}

Keywords: norms; emotion; arousal; valence; word lists.

O que é uma emoção e como ela influencia os nossos processos cognitivos? Eventos com carga emocional são processados diferentemente de eventos neutros? Quais processos de memória contribuem na lembrança de informações associadas a uma experiência emocional? As respostas para essas perguntas emergem do crescente número de estudos sobre a interação entre emoção e cognição (Oliva \& cols., 2006). Por exemplo, estudos apontam que informações emocionais são mais lembradas do que informações neutras (Kensinger \& Corkin, 2004), até mesmo em pacientes amnésicos (Sharot, Verfaellie \& Yonelinas, 2007). Apesar disso, os resultados na literatura sobre a interação entre emoção e cognição nem sempre concordam (Pessoa, McKenna, Gutierrez \& Ungerleider, 2002; Vuilleumier, Armory, Driver \& Dolan, 2001),

1 Este trabalho recebeu apoio financeiro da CAPES, sendo parte da Dissertação de Mestrado apresentada ao Programa de Pós-Graduação em Psicologia da Pontifícia Universidade Católica do Rio Grande do Sul pelo primeiro autor, sob a orientação da quarta autora, em 2007. Este trabalho também teve apoio do CNPq (301502/2006-4, 107804/2007-7). Os autores são gratos às sugestões de modificações feitas por dois revisores anônimos em versões anteriores deste manuscrito.

2 Endereço para correspondência: Pontifícia Universidade Católica do Rio Grande do Sul, Pós-Graduação em Psicologia. Av. Ipiranga, 6681 - Prédio 11 - sala 940. Porto Alegre, RS. CEP 90619-900. Fone/Fax: (51)3320-3633.E-mail: 1ilian@pucrs.br. possivelmente devido à falta de consenso sobre como definir, mensurar e manipular experimentalmente a variável emoção (Mauss \& Robinson, 2009).

Com o intuito de contribuir para que pesquisadores possam avançar na compreensão da influência da emoção na cognição, o objetivo do presente estudo foi obter medidas de emocionalidade para materiais verbais de idioma português-brasileiro. Para isso, descreveremos inicialmente as definições e medidas da variável emoção e, posteriormente, apresentaremos o material verbal para o qual as normas de emocionalidade foram coletadas.

\section{Emoção como Dimensões}

A subjetividade da experiência emocional sempre foi um obstáculo para a inserção da emoção como tópico de investigação científica (LeDoux, 2000). Para ultrapassar esse obstáculo, é preciso explicitar pelo menos dois tipos de definição relativas ao conceito de emoção: (a) uma definição que distingue emoção de outros fenômenos, como o humor; e (b) outra que indica o que mensurar para capturar o conceito de emoção, também conhecida como definição operacional.

No que diz respeito ao item $a$, a emoção pode ser caracterizada como um estado breve de prontidão para a ação frente 
a um estímulo específico. Por exemplo, ao nos depararmos com uma situação ameaçadora, reagimos enfrentando ou fugindo da situação. A emoção distingue-se do humor por este último ser caracterizado por um estado duradouro de disposição à ação, que não é específico a um estímulo (cf. Scherer, 2005, para outras distinções).

Em relação ao item $b$, é possível estudar a emoção humana a partir de algumas dimensões fundamentais (Bradley \& Lang, 1994; Lang, 1995; mas ver Mauss \& Robinson, 2009, para outras possibilidades). Segundo Lang, Bradley e Cuthbert (1999), a visão teórica que permite o estudo dimensional da emoção possui suas raízes no século XIX, já sendo defendida por Wundt (1896). No entanto, foi a partir das pesquisas realizadas por Osgood, Suci e Tannenbaum (1957) que a teoria dimensional da emoção se tornou uma alternativa científica para a mensuração de respostas emocionais. Osgood e cols. realizaram análises fatoriais em um grande número de descritores verbais emocionais associados a diversos tipos de estímulos (e.g., figuras, sons e palavras) e encontraram que a variância nas avaliações poderia ser explicada por duas dimensões principais: a valência (varia do prazer ao desprazer) e o alerta ${ }^{3}$ (varia da calma a estimulação). Além delas, os autores identificaram uma terceira dimensão, menos consistente que as anteriores, chamada de dominância (varia do controle à submissão). Posteriormente, Mehrabian e Russell (1974), utilizando outras escalas de diferencial semântico, chegaram a conclusões similares.

Seguindo os trabalhos precursores do estudo dimensional da emoção, Lang (1980) desenvolveu uma escala pictográfica não verbal para a avaliação de valência, alerta e dominância, chamada de Self-Assessment Manikin (SAM). Cada dimensão (i.e., valência e alerta) possui uma respectiva escala composta por cinco figuras (ver Figura 1). Os participantes avaliam a resposta emocional a um determinado estímulo assinalando uma das cinco figuras em cada escala, ou assinalando nos espaços entre as figuras. Portanto, cada escala é constituída por nove pontos. Desse modo, 9 é o escore máximo e 1 é o escore mínimo em cada dimensão. O escore médio é representado pela terceira figura, ou pelo escore 5 da escala. Kensinger e Corkin (2004) denominaram os itens que provocam reações emocionais classificadas com níveis baixos de valência (do escore 1 ao 3,99) de negativos, os com níveis médios (do escore 4 ao 6,99) foram denominados de neutros e os com níveis altos (do escore 7 ao 9) foram chamados de positivos. Quanto ao alerta, os itens classificados com níveis baixos (do escore 1 ao 5.99) foram descritos como não estimulantes ou relaxantes, e os com níveis altos (do escore 6 ao 9) foram chamados de estimulantes.

O SAM tem se mostrado um instrumento de boas qualidades psicométricas. As avaliações de valência e alerta do SAM correlacionam-se fortemente com a Escala do Diferencial Semântico de Mehrabian e Russell (1974), como indica o estudo comparativo de Bradley e Lang (1994). Além disso, as medidas do SAM apresentam alta correlação com respostas

3 Tradução usualmente utilizada na literatura brasileira para o termo arousal da língua inglesa (e.g., Ribeiro, Pompéia \& Bueno, 2004). fisiológicas (e.g., condutância elétrica da pele) (Bradley \& Lang, 1994). O SAM tem sido utilizado para a mensuração de respostas emocionais produzidas por palavras (Bradley \& Lang, 1999; Redondo, Fraga, Comesaña \& Perea, 2005) e imagens (Lang \& cols. 1999; Ribeiro, Pompéia \& Bueno, 2004). As escalas SAM têm contribuído para o estudo de questões fundamentais na compreensão da influência da emoção na atenção (Mather \& cols., 2004; Robinson, Storbeck, Meier, \& Kirkeby, 2004) e na memória (Hu, Stylos-Allan \& Walker, 2006; LaBar \& Phelps, 1998).

De forma geral, as principais vantagens do uso do SAM estão na sua fácil e ampla possibilidade de aplicação e no fato de propiciar uma rápida análise dos dados. Além disso, ele permite a realização de estudos interculturais em função de seu caráter pictórico, o que viabiliza comparações de bases de dados mais extensas (Ribeiro \& cols., 2004).

\section{O Paradigma Deese-Roediger-McDermott (DRM)}

Um tópico emergente no estudo do impacto da emoção na memória diz respeito a um tipo de distorção mnemônica denominada de falsas memórias (Payne, Elie, Blackwell \& Neuschatz, 1996; Santos \& Stein, 2008; Schacter \& Slotnick, 2004). O fenômeno das falsas memórias consiste na lembrança de eventos que nunca ocorreram (Brainerd \& Reyna, 2005) e os resultados experimentais sugerem que eventos negativos são mais suscetíveis ao falso reconhecimento do que eventos neutros (Marchewka \& cols., 2008) ou positivos (Brainerd, Stein, Silveira, Rohenkohl \& Reyna, 2008).

Dentre os procedimentos experimentais utilizados no estudo das falsas memórias, destaca-se o paradigma Deese-Roediger-McDermott (DRM). Esse paradigma foi criado por Roediger e McDermott (1995), baseado no trabalho de Deese (1959). Stein, Feix e Rohenkohl (2006) desenvolveram a versão brasileira desse procedimento, que consiste na apresentação de várias listas de 15 palavras semanticamente associadas a uma palavratema não apresentada na fase de estudo, denominada de palavra crítica. Por exemplo, todas as palavras da lista sorriso, felicidade, festa, vida, amigos, família, paz, nascimento, filhos, euforia, pular, diversão, sucesso, carnaval e gol são associadas semanticamente à palavra crítica alegria. Na fase de teste da memória, os participantes realizam um teste de recordação ou de reconhecimento com a presença do distrator crítico (nome dado à palavra crítica quando apresentada no teste de reconhecimento). O uso do procedimento DRM tem produzido altos índices de falsas recordações e falsos reconhecimentos (Karpicke, McCabe \& Roediger, 2008; Roediger, McDermott, Pisoni \& Gallo, 2004), caracterizados pela falsa lembrança da palavra crítica (e.g., Stein \& Pergher, 2001). Além disso, também permite a obtenção de índices de memória verdadeira.

Stein e Gomes (aceito) ressaltam que o uso de listas de palavras associadas não se limita apenas à investigação do fenômeno das falsas memórias. Como ele permite controle e manipulação de diversos fatores (e.g., associação semântica, frequência lexical, concretude e número de letras), as listas de palavras associadas podem ser utilizadas em tarefas de 


\section{Escala A - Valência}
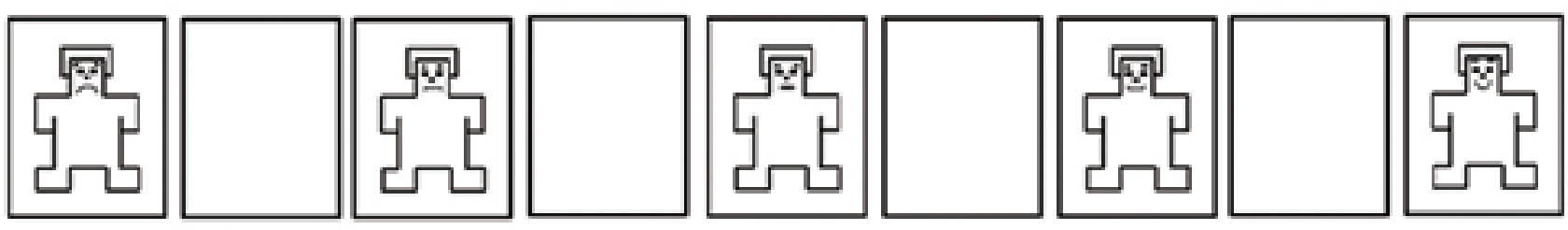

\section{Escala B - Alerta}
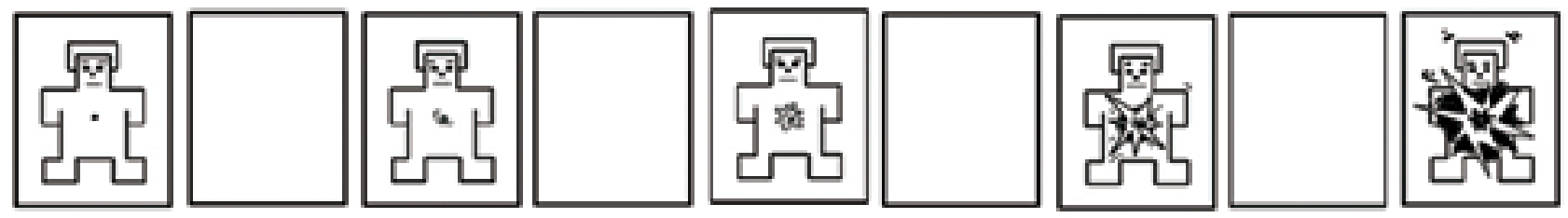

Figura 1. Escalas SAM para avaliação de valência (escala A) e alerta (escala B) (Lang, 1980).

decisão lexical, para investigação da memória implícita, tarefas de span de dígitos, para investigação da memória operacional, entre outras.

Nesse contexto, o objetivo do presente estudo foi obter índices de valência e alerta para cada uma das 44 listas de palavras associadas e suas palavras críticas da versão brasileira do DRM (Stein \& cols., 2006). Que saibamos, este é o primeiro estudo a realizar a normatização emocional desse tipo de material.

\section{Método}

A normatização dos índices de emocionalidade da versão brasileira do paradigma DRM incluiu duas coletas de dados distintas: (a) Estudo das listas de palavras - coleta de dados para as 44 listas de palavras associadas, sem a presença da palavra crítica; e (b) Estudo das palavras críticas - coleta de dados para as palavras críticas, sem a presença das listas.

\section{Participantes}

Os participantes de ambos os estudos foram constituídos por universitários de diversos cursos de graduação de uma universidade do interior do Rio Grande do Sul.

Estudo das listas de palavras. A coleta das normas de emocionalidade para as 44 listas contou com 431 participantes ( $23 \%$ homens), sendo que a idade variou de 18 a 45 anos $(M=23,85$ anos, $D P=6,89)$. Os participantes deste estudo foram divididos em quatro grupos, com a seguinte composição: 95, 107, 110 e 119.

Estudo das palavras críticas. A coleta relativa às palavras críticas envolveu 85 participantes ( $25,9 \%$ homens), com as idades variando de 18 a 42 anos $(M=24,58, D P=6,21)$.

\section{Instrumentos}

Para a avaliação da emocionalidade, os participantes de ambos os estudos utilizaram a versão lápis e papel do SAM (Lang, 1980), com escalas para julgar os estímulos tanto no que diz respeito à valência quanto ao alerta (ver Figura 1). Nessa escala, a valência pode ser avaliada desde desagradável (1) até agradável (9), sendo o alerta pontuado desde relaxado (1) até estimulado (9). Em ambas as escalas, um ponto intermediário (5) marca um setor neutro. Os estímulos utilizados nos estudos aqui descritos pertencem à versão brasileira do paradigma DRM (Stein \& cols., 2006)

Estudo das listas de palavras. Foram utilizados como estímulos 44 listas compostas por 15 palavras cada, totalizando 660 palavras. As 44 listas de palavras foram divididas em quatro blocos de 11 listas cada. Para controlar possíveis efeitos da ordem de apresentação das listas, foram criadas duas sequências aleatórias de apresentação dentro de cada bloco: ordem A e ordem B. As palavras das listas foram projetadas em uma tela, em letras maiúsculas, fonte Arial e tamanho 96, utilizando o programa Microsoft $\mathrm{B}$ PowerPoint $\AA$.

Estudo das palavras críticas. Foram utilizadas as 44 palavras críticas correspondentes às 44 listas de palavras associadas. Nesse caso, todas as palavras críticas foram apresentadas impressas no próprio material de coleta das avaliações.

\section{Procedimento}

Em ambos os estudos, os participantes foram testados coletivamente nas respectivas salas de aula. No primeiro momento, foi realizada uma apresentação da pesquisa e dos procedimentos éticos. Seguindo os procedimentos éticos de pesquisa com seres humanos, todos os participantes foram avisados sobre o caráter de participação voluntária e anônima. 
Os participantes que concordaram em fazer parte do estudo assinaram o Termo de Consentimento Livre e Esclarecido.

Estudo das listas de palavras. Aos participantes foram apresentados os objetivos e as instruções do experimento. Em seguida, foram distribuídas folhas-resposta para as avaliações, no formato SAM, da valência e alerta das listas de palavras. As instruções da tarefa foram semelhantes àquelas utilizadas por Lang e cols. (1999) e Ribeiro e cols. (2004), descritas abaixo:

Como vocês podem observar, as folhas-resposta contêm duas escalas distribuidas em linhas. Cada linha contém dois conjuntos de cinco desenhos. A primeira escala (escala A) (situada no lado esquerdo do papel) avalia o quanto a lista de palavras lhe pareceu agradável ou desagradável. Por exemplo, se a lista de palavras apresentada lhe pareceu muito agradável, marque um X sobre o desenho mais à direita. Se a lista apresentada lhe pareceu desagradável, marque um X sobre o desenho mais à esquerda. Se a lista não lhe pareceu nem totalmente agradável nem totalmente desagradável, marque um X sobre o desenho que melhor corresponda à sua sensação. A segunda escala (escala $B$ ) avalia o quanto a lista de palavras the deixou relaxado ou estimulado. Se a lista lhe deixou muito relaxado, marque um $X$ sobre o desenho da esquerda. Se a lista lhe deixou muito estimulado, marque um X sobre o desenho da direita. Se a lista não lhe deixou nem totalmente relaxado nem totalmente estimulado, marque um X sobre o desenho que melhor corresponda à sua sensação. As duas avaliações são independentes e você pode marcar qualquer ponto da escala sem considerar o que já marcou na escala anterior. Assim, pode acontecer de alguma lista ser avaliada como desagradável e também como estimulante. Vocês devem observar que se trata de uma avaliação subjetiva, não existindo respostas certas ou erradas. Alguma dúvida?

Após as instruções iniciais, foi realizada uma fase de treino para nos assegurarmos de que os participantes haviam entendido as instruções. Terminado o treino e esclarecidas as dúvidas, foram apresentadas as listas de palavras a serem avaliadas. Cada bloco foi apresentado a um dos quatro grupos de participantes. Portanto, cada participante avaliou apenas um bloco de 11 listas de palavras.

A organização da apresentação das listas é descrita a seguir. Antes da apresentação de cada lista, foi dada a seguinte instrução: "Prepara-te para avaliar a lista". Após a apresentação de cada uma das listas, sendo uma palavra por vez (cada palavra ficava exposta na tela por 2 segundos), foi apresentada uma lâmina contendo a instrução: "Avalie a escala A", que era também lida para os participantes. Na sequência, foi mostrada uma nova lâmina também acompanhada de instrução oral: "Avalie a escala B”. Em seguida, foi apresentada uma lâmina em branco. Esse procedimento foi repetido de maneira sucessiva ao longo das 11 listas e foi idêntico entre os blocos. O tempo total de realização da coleta de dados foi de aproximadamente 30 minutos.

Estudo das palavras críticas. Inicialmente, os participantes foram avisados sobre o objetivo e as instruções do experimento. Em seguida, foi entregue uma folha-resposta para cada participante. Essa folha continha 44 palavras críticas que foram impressas juntamente com as escalas de avaliação (SAM) de valência e alerta. Além disso, foi adicionado um exemplo no início da folha, sendo este utilizado durante a fase de treino. As instruções utilizadas neste estudo foram as mesmas instruções iniciais do "estudo das listas de palavras", apenas substituindo os termos "lista de palavras" e "lista" pelo termo "palavra".

Neste estudo, a tarefa dos participantes consistiu em ler a palavra crítica e realizar a avaliação de valência e alerta dela nas escalas SAM, situadas logo abaixo da palavra crítica. O tempo de realização dessa tarefa foi de aproximadamente 15 minutos.

\section{Resultados e Discussão}

A emocionalidade média de cada lista de palavras associadas foi calculada com base em uma amostra de pelo menos 95 e, no máximo, 119 avaliações. Já a emocionalidade média de cada palavra crítica foi calculada a partir de uma amostra de 85 avaliações. As tabelas 1 e 2 apresentam as médias e desvios-padrão de valência e alerta, em ordem decrescente das médias da lista, obtidos para cada uma das 44 listas de palavras e suas palavras críticas da versão brasileira do paradigma DRM.

A percepção emocional dos estímulos provocada pelas listas e palavras críticas pode ser representada graficamente por sua localização em um espaço bidimensional, denominado de "espaço afetivo" (Russell, 1980). O espaço afetivo é constituído pela dimensão valência (ordenada) e pela dimensão alerta (abscissa). A Figura 2 ilustra o espaço afetivo das 44 listas de palavras e palavras críticas.

Assim como ocorre em estudos de normatização da emocionalidade para fotografias (Lang \& cols., 1999) e de estímulos acústicos e verbais (Bradley, Zack \& Lang, 1994), não encontramos listas de palavras em todos os quadrantes do espaço afetivo. As listas desagradáveis tendem a se agrupar no quadrante inferior direito do gráfico. Esse resultado concorda com a experiência cotidiana, já que eventos que nos desagradam geralmente provocam estimulação (e.g., uma pessoa dificilmente irá sentir-se relaxada ao presenciar um assalto ou uma batida de carro). Assim, por exemplo, a lista da palavra raiva foi avaliada com uma valência média baixa $(1,6)$ e um alerta elevado $(6,51)$. No entanto, as listas de palavras agradáveis (parte superior do gráfico) se distribuem praticamente por toda a extensão do eixo das abscissas. Assim, encontramos listas de palavras agradáveis como dormir (valência $=7,41$ ) classificadas com valores baixos de alerta $(3,55)$, enquanto outras listas agradáveis como paixão (valência=8,24) são avaliadas com um valor elevado de alerta $(7,44)$. Já as listas de palavras de valência intermediária ou neutra foram avaliadas com um nível de alerta intermediário (e.g., borracha, valência=5,1; alerta $=4,51)$. Esse resultado também obtém apoio da experiência cotidiana, pois é incomum que um estímulo de valência neutra nos estimule ou relaxe. $\mathrm{O}$ mesmo padrão é observado para o espaço afetivo das palavras críticas. Apesar de terem sido observados valores próximos aos 
Tabela 1. Médias e desvios-padrão de valência para as 44 listas de palavras e suas palavras críticas.

\begin{tabular}{|c|c|c|c|c|}
\hline \multirow{3}{*}{ Nome da lista } & \multicolumn{4}{|c|}{ Valência } \\
\hline & \multicolumn{2}{|c|}{ Lista } & \multicolumn{2}{|c|}{ Palavra crítica } \\
\hline & $M$ & $(D P)$ & $M$ & $(D P)$ \\
\hline Fruta & 8,66 & $(0,97)$ & 8,12 & $(1,27)$ \\
\hline Alegria & 8,62 & $(1,30)$ & 8,79 & $(0,73)$ \\
\hline Macio & 8,54 & $(1,45)$ & 8,28 & $(1,37)$ \\
\hline Satisfação & 8,52 & $(1,35)$ & 8,61 & $(1,18)$ \\
\hline Música & 8,26 & $(1,88)$ & 8,64 & $(0,92)$ \\
\hline Paixão & 8,24 & $(1,35)$ & 8,39 & $(1,60)$ \\
\hline Rio & 8,03 & $(2,05)$ & 7,38 & $(2,33)$ \\
\hline Menina & 8,01 & $(1,91)$ & 7,29 & $(2,05)$ \\
\hline Janela & 7,71 & $(1,82)$ & 7,09 & $(1,84)$ \\
\hline Alívio & 7,66 & $(1,88)$ & 8,62 & $(0,79)$ \\
\hline Montanha & 7,62 & $(2,19)$ & 7,21 & $(2,01)$ \\
\hline Pão & 7,59 & $(1,96)$ & 8,04 & $(1,64)$ \\
\hline Dormir & 7,41 & $(1,92)$ & 8,33 & $(1,31)$ \\
\hline Cadeira & 7,10 & $(1,79)$ & 6,22 & $(2,18)$ \\
\hline Homem & 7,08 & $(1,96)$ & 6,95 & $(2,25)$ \\
\hline Camisa & 6,90 & $(2,12)$ & 6,39 & $(1,89)$ \\
\hline Carro & 6,61 & $(2,22)$ & 8,26 & $(1,18)$ \\
\hline Frio & 6,50 & $(2,63)$ & 3,44 & $(2,78)$ \\
\hline Bandeira & 6,43 & $(2,27)$ & 6,01 & $(2,17)$ \\
\hline Cidade & 6,43 & $(2,36)$ & 7,00 & $(2,02)$ \\
\hline Doutor & 6,41 & $(2,17)$ & 4,84 & $(2,25)$ \\
\hline Doce & 6,41 & $(2,01)$ & 8,31 & $(1,39)$ \\
\hline
\end{tabular}

\begin{tabular}{|c|c|c|c|c|}
\hline \multirow{3}{*}{ Nome da lista } & \multicolumn{4}{|c|}{ Valência } \\
\hline & \multicolumn{2}{|c|}{ Lista } & \multicolumn{2}{|c|}{ Palavra crítica } \\
\hline & $M$ & $(D P)$ & $M$ & $(D P)$ \\
\hline Xícara & 6,21 & $(2,39)$ & 6,55 & $(2,04)$ \\
\hline Rei & 6,06 & $(2,77)$ & 5,04 & $(2,42)$ \\
\hline Caneta & 5,61 & $(2,38)$ & 6,61 & $(1,99)$ \\
\hline Cheirar & 5,33 & $(2,20)$ & 6,32 & $(2,26)$ \\
\hline Alto & 5,13 & $(1,87)$ & 5,29 & $(2,59)$ \\
\hline Borracha & 5,10 & $(2,27)$ & 5,56 & $(2,03)$ \\
\hline Leão & 4,78 & $(2,39)$ & 4,09 & $(2,55)$ \\
\hline Pé & 4,72 & $(2,19)$ & 5,72 & $(2,03)$ \\
\hline Áspero & 4,12 & $(2,22)$ & 2,46 & $(1,67)$ \\
\hline Agulha & 3,99 & $(2,17)$ & 2,71 & $(1,90)$ \\
\hline Exército & 3,75 & $(2,53)$ & 5,73 & $(2,51)$ \\
\hline Devagar & 2,92 & $(2,10)$ & 4,00 & $(2,34)$ \\
\hline Lixo & 2,34 & $(1,94)$ & 1,35 & $(0,90)$ \\
\hline Preto & 2,25 & $(1,80)$ & 6,09 & $(2,16)$ \\
\hline Culpa & 2,03 & $(1,58)$ & 1,48 & $(1,30)$ \\
\hline Mágoa & 1,70 & $(1,70)$ & 1,19 & $(0,61)$ \\
\hline Fumar & 1,61 & $(1,67)$ & 1,98 & $(2,15)$ \\
\hline Raiva & 1,60 & $(1,54)$ & 1,66 & $(1,79)$ \\
\hline Dor & 1,57 & $(1,50)$ & 1,31 & $(0,95)$ \\
\hline Aranha & 1,56 & $(1,35)$ & 1,88 & $(1,32)$ \\
\hline Ladrão & 1,27 & $(0,94)$ & 1,16 & $(0,55)$ \\
\hline Medo & 1,20 & $(0,70)$ & 1,72 & $(1,39)$ \\
\hline
\end{tabular}

Nota: $M=$ média, $D P=$ desvio-padrão.

extremos da escala de valência, não foram observados valores próximos aos extremos na escala de alerta. Esse resultado concorda com o achado de que palavras, se comparadas a fotografias, tendem a produzir avaliações menos extremas de alerta (Redondo \& cols., 2005).

A confiabilidade interna das normas para as 44 listas de palavras foi calculada por meio de correlações de Pearson entre as avaliações médias das listas de palavras associadas, de cada ordem de apresentação (A e B). Se a ordem de apresentação influenciou a avaliação de valência e alerta das listas, então é esperada uma correlação baixa ou inexistente entre as ordens A e B. Essa análise mostrou os seguintes resultados: $r=0,98(p<0,001)$ para a valência e $r=0,74(p<0,001)$ para o alerta. Assim, essas correlações indicam que a ordem de apresentação não parece ter influenciado nas avaliações de valência e alerta das listas.
Para a avaliação da confiabilidade interna das normas para as 44 palavras críticas, compararam-se as avaliações por dois subgrupos de participantes, obtidos por meio de uma separação aleatória do conjunto total dos 85 componentes da amostra. Após a obtenção das médias de avaliações dos subgrupos para cada uma das palavras críticas, calcularamse correlações de Pearson entre essas médias, sendo $r=0,99$ $(p<0,001)$ para a valência e $r=0,87(p<0,001)$ para o alerta. Esses dados apontam para uma boa consistência interna das normas relativas às palavras críticas. Igualmente, observaram-se correlações positivas entre as avaliações das listas e de suas palavras críticas. Quer dizer, à medida que as avaliações de valência e alerta da lista aumentam, estas são acompanhadas pelo aumento das avaliações de valência e alerta da sua palavra crítica $\left(r_{\text {valência }}=0,90 ; p<0,001\right)$ $\left(r_{\text {alerta }}=0,50 ; p<0,001\right)$. 
Tabela 2. Médias e desvios-padrão de alerta para as 44 listas de palavras e suas palavras críticas.

\begin{tabular}{|c|c|c|c|c|}
\hline \multirow{3}{*}{ Nome da lista } & \multicolumn{4}{|c|}{ Alerta } \\
\hline & \multicolumn{2}{|c|}{ Lista } & \multicolumn{2}{|c|}{ Palavra crítica } \\
\hline & $M$ & $(D P)$ & $M$ & $(D P)$ \\
\hline Paixão & 7,44 & $(2,32)$ & 6,67 & $(3,16)$ \\
\hline Alegria & 7,18 & $(2,89)$ & 5,73 & $(3,49)$ \\
\hline Medo & 6,82 & $(2,38)$ & 6,81 & $(2,58)$ \\
\hline Montanha & 6,76 & $(2,63)$ & 5,16 & $(2,98)$ \\
\hline Carro & 6,67 & $(2,24)$ & 5,55 & $(3,23)$ \\
\hline Cidade & 6,59 & $(2,21)$ & 5,78 & $(2,60)$ \\
\hline Raiva & 6,51 & $(2,47)$ & 6,86 & $(2,70)$ \\
\hline Satisfação & 6,44 & $(3,30)$ & 4,99 & $(3,52)$ \\
\hline Ladrão & 6.37 & $(2,59)$ & 7,01 & $(2,68)$ \\
\hline Rei & 6.33 & $(2,34)$ & 5,11 & $(2,26)$ \\
\hline Culpa & 6.30 & $(2,34)$ & 6,38 & $(2,49)$ \\
\hline Doutor & 6.05 & $(2,35)$ & 4,44 & $(2,37)$ \\
\hline Aranha & 6.04 & $(2,41)$ & 6,26 & $(2,56)$ \\
\hline Bandeira & 5.92 & $(2,32)$ & 5,05 & $(2,06)$ \\
\hline Caneta & 5.83 & $(2,17)$ & 5,02 & $(2,47)$ \\
\hline Dor & 5.78 & $(2,57)$ & 2,71 & $(2,93)$ \\
\hline Música & 5.69 & $(3,43)$ & 5,07 & $(3,55)$ \\
\hline Doce & 5.68 & $(2,42)$ & 5,08 & $(3,34)$ \\
\hline Fruta & 5.59 & $(3,29)$ & 4,59 & $(2,90)$ \\
\hline Leão & 5.53 & $(2,26)$ & 6,91 & $(2,30)$ \\
\hline Lixo & 5.52 & $(2,08)$ & 5,66 & $(2,81)$ \\
\hline Homem & 5.49 & $(2,51)$ & 6,16 & $(2,73)$ \\
\hline
\end{tabular}

\begin{tabular}{|c|c|c|c|c|}
\hline \multirow{3}{*}{ Nome da lista } & \multicolumn{4}{|c|}{ Alerta } \\
\hline & \multicolumn{2}{|c|}{ Lista } & \multicolumn{2}{|c|}{ Palavra crítica } \\
\hline & $M$ & $(D P)$ & $M$ & $(D P)$ \\
\hline Mágoa & 5,45 & $(2,47)$ & 6,07 & $(3,02)$ \\
\hline Exército & 5,44 & $(2,46)$ & 5,44 & $(2,56)$ \\
\hline Pão & 5,41 & $(2,94)$ & 5,02 & $(2,82)$ \\
\hline Fumar & 5,41 & $(2,39)$ & 5,19 & $(2,89)$ \\
\hline Preto & 5,29 & $(2,31)$ & 5,19 & $(2,29)$ \\
\hline Agulha & 5,07 & $(2,03)$ & 5,93 & $(2,36)$ \\
\hline Áspero & 4,99 & $(2,02)$ & 5,22 & $(2,34)$ \\
\hline Alto & 4,88 & $(1,62)$ & 6,47 & $(2,21)$ \\
\hline Camisa & 4,83 & $(2,67)$ & 4,11 & $(2,16)$ \\
\hline Pé & 4,72 & $(1,89)$ & 4,56 & $(1,95)$ \\
\hline Xícara & 4,67 & $(2,59)$ & 3,91 & $(2,61)$ \\
\hline Devagar & 4,58 & $(2,65)$ & 4,09 & $(2,45)$ \\
\hline Cheirar & 4,57 & $(2,23)$ & 5,76 & $(2,28)$ \\
\hline Borracha & 4,51 & $(2,04)$ & 4,76 & $(2,03)$ \\
\hline Rio & 4,29 & $(3,31)$ & 5,07 & $(3,29)$ \\
\hline Frio & 4,10 & $(2,82)$ & 4,84 & $(2,70)$ \\
\hline Menina & 4,08 & $(3,02)$ & 4,79 & $(2,94)$ \\
\hline Alívio & 4,05 & $(2,74)$ & 3,18 & $(3,02)$ \\
\hline Cadeira & 4,00 & $(2,50)$ & 3,51 & $(2,12)$ \\
\hline Janela & 3,97 & $(3,05)$ & 4,15 & $(2,43)$ \\
\hline Dormir & 3,55 & $(2,71)$ & 6,18 & $(2,80)$ \\
\hline Macio & 3,26 & $(3,22)$ & 3,65 & $(3,25)$ \\
\hline
\end{tabular}

Nota: $M=$ média, $(D P)=$ desvio-padrão.

\section{Considerações Finais}

O objetivo do presente estudo foi obter índices de valência e alerta para cada uma das 44 listas de palavras associadas e suas palavras críticas da versão brasileira do DRM (Stein \& cols., 2006). As tabelas aqui disponibilizadas fornecem uma fonte para seleção de estímulos a serem utilizados em pesquisas sobre emoção e cognição. Além disso, os resultados indicaram boa confiabilidade interna das medidas de valência e alerta, tanto para as listas de palavras quanto para as palavras críticas. Dessa forma, com a intenção de que pesquisadores brasileiros possam avançar na compreensão da influência da emoção na cognição, o presente estudo contribuiu ao fornecer estímulos verbais para o desenvolvimento de novas pesquisas nessa área.

Devemos salientar, no entanto, que a normatização foi realizada apenas para a população de estudantes universitários, tal como ocorre em grande parte dos estudos de normatização emocional de estímulos (e.g., Bradley \& Lang, 1999), de modo que o material deve ser utilizado com essa ressalva para outras populações. Por esse motivo, sugerimos que estudos futuros contemplem a avaliação da emocionalidade com amostras diferentes, tais como idosos e grupos clínicos.

\section{Referências}

Bradley, M. M., \& Lang, P. J. (1994). Measuring emotion: The self-assessment manikin and the semantic differential. Journal of Behavioral Therapy and Experimental Psychiatry, 25, 49-59.

Bradley, M. M., \& Lang, P. J. (1999). Affective norms for English words (ANEW). Gainesville, FL: The National Institute of Mental Health Center for The Study of Emotion and Attention, University of Florida. 

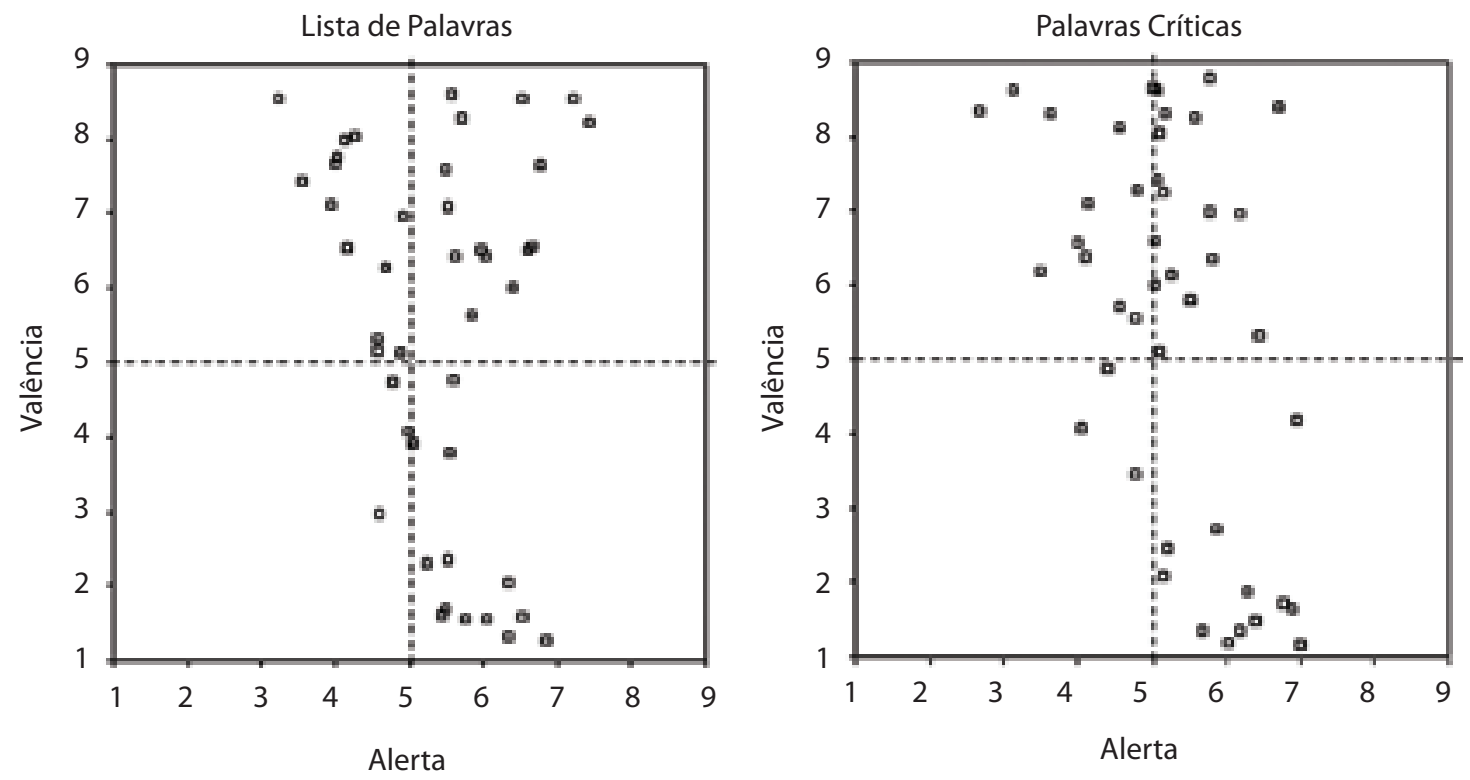

Figura 2. Espaço afetivo das 44 listas de palavras e palavras críticas.

Bradley, M. M., Zack, J., \& Lang, P. J. (1994). Cries, screams, and shouts of joy: Affective responses to environmental sounds [Abstract]. Psychophysiology, 31, (Suppl. 1), S29.

Brainerd, C. J., \& Reyna, V. F. (2005). The science of false memory. New York: Oxford University Press.

Brainerd, C. J., Stein, L. M., Silveira, R. A. T., Rohenkohl, G., $\&$ Reyna, V. F. (2008). How does negative emotion induce false memories? Psychological Science, 19, 919-925.

Deese, J. (1959). On the prediction of occurrence of particular verbal intrusions in immediate recall. Journal of Experimental Psychology, 58, 17-22.

Hu, P., Stylos-Allan, M., \& Walker, M. P. (2006). Sleep facilitates consolidation of emotional declarative memory. Psychological Science, 17, 891-898.

Karpicke, J. D., McCabe, D. P., \& Roediger, H. L. (2008). False memories are not suprising: The subjective experience of an associative memory illusion. Journal of Memory and Language, $58,1065-1079$.

Kensinger, E. A., \& Corkin, S. (2004). Two routes to emotional memory: Distinct neural processes for valence and arousal. Proceedings of the National Academy of Sciences of the USA, 101, 3310-3315.

LaBar, K. S., \& Phelps, E. A. (1998). Arousal-mediated memory consolidation: Role of the medial temporal lobe in humans. Psychological Science, 9, 490-493.

Lang, P. J. (1980). Behavioral treatment and bio-behavioral assessment: Computer applications. Em J. B. Sidowski, J. H. Johnson \& T. A. Williams (Eds.), Technology in mental health care delivery systems (pp. 119-137). Norwood: Ablex.

Lang, P. J., Bradley, M. M., \& Cuthbert, B. N. (1999). International affective picture system (IAPS): Technical manual and affective ratings. Gainesville, FL: The Center for Research in Psychophysiology, University of Florida.

Lang, P. J. (1995). The emotion probe. American Pychologist, 50, 372-385.

LeDoux, J. E. (2000). Emotion circuits in the brain. Annual Review of Neuroscience, 23, 155-84.
Marchewka, A., Brechmann, A., Nowicka, A., Jednoróg, K. Scheich, H., \& Grabowska, A. (2008). False recognition of emotional stimuli is lateralised in the brain: An fMRI study. Neurobiology of Learning and Memory, 90, 280-284.

Mather, M., Canli, T., English, T., Whitfield, S., Wais, P., Ochsner, K., Gabrieli, J. D. E., \& Cartensen, L. L. (2004). Amygdala responses to emotionally valenced stimuli in older and younger adults. Psychological Science, 15, 259-263.

Mauss, I. B., \& Robinson, M. D. (2009). Measures of emotion: A review. Cognition \& Emotion, 23, 209-237.

Mehrabian, A., \& Russell, J. A. (1974). An approach to environmental psychology. Cambridge: MIT Press.

Oliva, A. D., Otta, E., Ribeiro, F. L., Bussab, V. S. R., Lopes, F. A., Yamamoto, M. E., \& Moura, M. L. S. (2006). Razão, emoção e ação em cena: a mente humana sob um olhar evolucionista. Psicologia: Teoria e Pesquisa, 22, 53-62.

Osgood, C., Suci, G., \& Tannenbaum, P. (1957). The measurement of meaning. Urbana: University of Ilinois Press.

Payne, D. G., Elie, C. J., Blackwell, J. M., \& Neuschatz, J. S. (1996). Memory illusions: Recalling, recognizing, and recollecting events that never occurred. Journal of Memory and Language, 35, 261-285.

Pessoa, L., McKenna, M., Gutierrez, E., Ungerleider, L. G. (2002). Neural processing of emotional faces requires attention. Proceedings of the National Academy of Sciences of the USA, 99, 11458-11463.

Redondo, J., Fraga, I., Comesaña, M., \& Perea, M. (2005). Estudio normativo del valor afectivo de 478 palabras españolas. Psicológica, 26, 317-326.

Ribeiro, R. L., Pompéia, S., \& Bueno, O. F. A. (2004). Normas brasileiras para o International Affective Picture System (IAPS): comunicação breve. Revista de Psiquiatria do Rio Grande do Sul, 26, 190-194.

Robinson, M. D., Storbeck, J., Meier, B. P., \& Kirkeby, B. S. (2004). Wacth out! That could be dangerous: Valence-arousal interaction in evaluative processing. Personality and Social Psychology Bulletin, 30, 1472-1484. 
Roediger, H. L., \& McDermott, K. B. (1995). Creating false memories: Remembering words not presented on lists. Journal of Experimental Psychology: Learning, Memory, and Cognition, 21, 803-814.

Roediger, H. L., McDermott, K. B., Pisoni, D. B., \& Gallo, D. A. (2004). Illusory recollection of voices. Memory, 12, 586-602.

Russell, J. A. (1980). A circumplex model of affect. Journal of Personality and Social Psychology, 39, 1161-1178.

Santos, R. F., \& Stein, L. M. (2008). A influência das emoções nas falsas memórias: uma revisão crítica. Psicologia USP, 19, 415-434.

Schacter, D. L., \& Slotnick, S. D. (2004). The cognitive neuroscience of memory distortion. Neuron, 44, 149-160.

Scherer, K. R. (2005). What are emotions? And how can they be measured? Social Science Information, 44, 695-729.

Sharot, T., Verfaellie, M., \& Yonelinas, A. P. (2007). How emotion strengthens the recollective experience: A time-dependent hippocampal process. PLoS ONE, 2, e1068.

Stein, L. M., \& Gomes, C. F. A. (aceito). Normas brasileiras para listas de palavras associadas: associação semântica, concretude, frequência e emocionalidade. Psicologia: Teoria e Pesquisa.
Stein, L. M., \& Pergher, G. K. (2001). Criando falsas memórias em adultos por meio de palavras associadas. Psicologia: Reflexão e Crítica, 14, 353-366.

Stein, L. M., Feix, L. F., \& Rohenkohl, G. (2006) Avanços metodológicos no estudo das falsas memórias: construção e normatização do Procedimento da Lista de Palavras Associadas à realidade brasileira. Psicologia: Reflexão e Crítica, 19, 166-176.

Vuilleumier, P., Armony, J. L., Driver, J., \& Dolan, R. J. (2001). Effects of attention and emotion on face processing in the human brain: An event-related fMRI study. Neuron, 30, 829-841.

Wundt, W. (1896). Compendio de Psicologia. Madrid: La España Moderna.
Recebido em 01.11.07

Versão final em 24.04.09

Aceito em 08.06.09 